\title{
Engineering Energy-Efficient Target Detection Applications in Wireless Sensor Networks
}

\author{
Paolo Medagliani* ${ }^{*}$, Jérémie Leguay ${ }^{\dagger}$, Vincent Gay ${ }^{\dagger}$, Mario Lopez-Ramos ${ }^{\dagger}$ and Gianluigi Ferrari* \\ ${ }^{*}$ Wireless Ad-hoc and Sensor Networks (WASN) Laboratory, University of Parma, viale G.P. Usberti 181/A, Parma, Italy \\ Emails: \{paolo.medagliani, gianluigi.ferrari\}@unipr.it \\ †Thales Communications, 160 Bd de Valmy, Colombes Cedex, France \\ Emails: \{paolo.medagliani, jeremie.leguay, vincent.gay, mario.lopezramos\}@fr.thalesgroup.com
}

\begin{abstract}
This paper addresses the problem of engineering energy-efficient target detection applications using unattended Wireless Sensor Networks (WSNs) for long-lasting surveillance of areas of interest. As battery energy depletion is an issue in this context, an approach consists of switching on and off sensing and communication modules of wireless sensors according to duty cycles. Making these modules work in an intermittent fashion impacts (i) the latency of notification transmission (depending on the communication duty cycle) and (ii) the probability of missed target detection (depending on the number of deployed nodes and the sensing duty cycle). In order to optimize the system parameters according to performance objectives, we first derive an analytical engineering toolkit which evaluates the probability of missed detection $\left(P_{\mathrm{md}}\right)$, the notification transmission latency $(D)$, and the network lifetime $(\mathcal{L})$ under the assumption of random node deployment. Then, we show how this toolbox can be used to optimally configure system parameters under realistic performance constraints.
\end{abstract}

\section{INTRODUCTION}

Wireless Sensor Networks (WSNs) are formed by batterypowered devices commonly used for environmental monitoring, military surveillance, and industrial automation. These devices are typically composed of an embedded microcontroller with some memory, a radio transceiver, physical transducers that sense the environment, and a battery. Recent advances in hardware miniaturization, low-power radio communications, and battery lifetime, together with the increasing affordability of such devices, are paving the road for a widespread usage of WSNs in a vast array of applications.

WSNs are expected to create a major shift in future pervasive computing applications, enabling a link between information systems and physical environments. In particular, in vast areas, traditional detection systems, such as cameras, fail to provide relevant information, whereas hundreds of tiny sensors running on batteries for months, or even years, can provide data about the environment, detect and locate events as well as trigger actuators. For instance, in scenarios such as a natural reserve, a large number of affordable sensor nodes with seismic and acoustic sensors can be spread throughout the area to study environmental conditions and animal behaviors unintrusively (i.e., without attaching devices to animals), to attract animals through sounds, to enable interactive applications, such as guiding visitors to animal herds, as well as to protect endangered species from illegal hunters. In these contexts, the whole application functionality relies on the ability to efficiently detect any incoming target (e.g., animal, hunter, etc.) in a given area of interest.

This paper addresses the problem of target detection using a long-term deployment of WSN nodes in a large monitored area. In such vast and long-term deployments, one of the main design goals is to maximize the operational lifetime of the system-typically by periodically switching off the sensing and communication parts-while ensuring that targets will eventually be detected and that the corresponding notification will be transmitted within a given time interval. This paper proposes an analytical framework to characterize the performance of the network in terms of the probability of missing a target, latency of notification transmission, and average energy consumption. In addition, the paper presents an engineering toolbox to efficiently set the configuration parameters in order to make a WSN function at a desired operating point, characterized by a trade-off between energy consumption and quality of service (in terms of detection capabilities and latency). We then validate the use of our toolbox to optimally configure a given WSN under realistic constraints.

In the literature, a few papers address in detail the problem of target detection and decision reporting. In [1], the authors present the design and the implementation of a monitoring system, referred to as VigilNet, based on a WSN. The authors derive an energy-efficient adaptable surveillance strategy and validate it through experimental tests. In [2], under the assumptions that the road network map is known and the target movement is confined into roads, the authors describe an algorithm, referred to as Virtual Scanning Algorithm, which ensures that the incoming target will be detected before reaching a given protection point. However, the above approaches do not provide a global analytical framework for the optimal tuning of system parameters, such as sensing and communication duty cycles.

This paper is structured as follows. In Section II, we describe the problem and the simulation set-up. In Section III, we present the analytical framework for determining the probability of missed detection, together with simulation results, which confirm its validity. Sections IV and V provide analytical models for the derivation of the latency and the energy consumption. In Section VI, we show how to configure a WSN using our analytical toolbox. Finally, Section VII 
concludes the paper.

\section{SCENARIO OF INTEREST}

This section first describes the target detection problem addressed in this paper. Then, it introduces the reference WSN system model that will be considered in the rest of the paper.

\section{A. The Target Detection Problem}

The surveillance of a given area is important in many military and civilian applications. In particular, a Wireless Sensor Network (WSN) can help detect an incoming target which crosses the monitored area. Upon the detection of a target, an alert message is sent to a gateway node, namely the Access Point (AP), which can reach control centers outside the network. We characterize the operating point of a WSN by a triplet of performance indicators, including the probability of missed detection $\left(P_{\mathrm{md}}\right)$, the notification transmission latency $(D)$, and the network lifetime $(\mathcal{L})$.

The problem of target detection using a long-term deployment of WSN nodes lies in the unavoidable trade-offs that energy saving strategies raise in terms of detection capability, responsiveness, and network lifetime. In these battery-powered networks, nodes are cyclically "switched off," according to proper duty cycles, generally at both sensing and the communication levels, in order to save energy. However, while extending the network lifetime, these operations also have an impact on (i) $P_{\mathrm{md}}$, since a node may be off when the target crosses its sensed area, and (ii) $D$, since asleep nodes can slow down the transmission of an alert message towards the AP.

The goal of this paper is first to provide an analytical framework for examining the performance indicators that characterize surveillance systems using a WSN. Second, considering a number of pre-defined operational constraints, the paper aims at engineering toolkit for tuning system-wide parameters, such as sensing and communication duty cycles, in order to find the optimal operating point which maximizes the network lifetime and the probability of detection, and also minimizes the latency.

\section{B. WSN Model}

The main parameters of the overall system model are listed in Table I. The default values presented in this table have been taken from the data-sheet of a commercial WSN node. The wireless sensor devices considered in this paper embed two main sub-units, i.e., (i) the sensing sub-unit and (ii) the communication sub-unit. The former is equipped with a seismic sensor, whose sensing range $r_{\mathrm{s}}$ is greatest over a rocky surface, in which the vibrations due to an incoming target propagate with low attenuation. Since seismic sensors can be placed over different surfaces, such as sandy or clayey terrains, where the propagation model is different and the attenuation is higher, we consider different values of $r_{\mathrm{s}}$. In order to reduce the energy consumption of the system, the sensing part can be periodically switched off, according to a normalized duty cycle $\beta_{\text {sens }} \in[0,1]$ over a period $t_{\text {sens }}$ (dimension: [s]). More precisely, nodes sense the surrounding

\begin{tabular}{|l|c|c|}
\hline \multicolumn{3}{|c|}{ Constants } \\
\hline \hline Side of monitored area & $d_{\mathrm{s}}$ & $1000 \mathrm{~m}$ \\
\hline Speed of the target & $v$ & $15 \mathrm{~m} / \mathrm{s}$ \\
\hline \hline Sensing power consumption & $\Omega_{\mathrm{sens}}$ & $0.0036 \mathrm{~W}$ \\
\hline \hline Communication period & $t_{\text {comm }}$ & $106 \mathrm{~ms}$ \\
\hline Transmission range & $r_{\mathrm{T}}$ & $250 \mathrm{~m}$ \\
\hline Preamble duration & $S_{\mathrm{p}}$ & $0.26 \mathrm{~ms}$ \\
\hline Ack window duration & $S_{\mathrm{al}}$ & $0.26 \mathrm{~ms}$ \\
\hline Packet duration & $S_{\mathrm{d}}$ & $0.93 \mathrm{~ms}$ \\
\hline Transmission power consumption & $\Omega_{\mathrm{T}_{\mathrm{x}}}$ & $0.0511 \mathrm{~W}$ \\
\hline Reception power consumption & $\Omega_{\mathrm{R}_{\mathrm{x}}}$ & $0.0588 \mathrm{~W}$ \\
\hline Sleep power consumption & $\Omega_{\mathrm{s}}$ & $2.4 \cdot 10^{-7} \mathrm{~W}$ \\
\hline \hline \multicolumn{2}{|c|}{ Variables (with default values) } \\
\hline \hline Number of nodes in the network & $\mathrm{N}$ & 50 \\
\hline Sensing range of each node & $r_{\mathrm{s}}$ & $20,35,50 \mathrm{~m}$ \\
\hline Average number of hops & $N_{\mathrm{hop}}$ & 3 \\
\hline \hline Sensing duty cycle & $\beta_{\mathrm{sens}}$ & $0.1-1$ \\
\hline Sensing period & $t_{\mathrm{sens}}$ & $15 \mathrm{~s}$ \\
\hline \hline Communication duty cycle & $\beta_{\mathrm{comm}}$ & $0.0025-1$ \\
\hline
\end{tabular}

Table I

CONSTANTS AND VARIABLES CONSIDERED.

environment for an interval of length $\beta_{\text {sens }} t_{\text {sens }}$ and sleep for an interval of duration $\left(1-\beta_{\text {sens }}\right) t_{\text {sens. }}$. The power consumption associated with the sensing operations is denoted as $\Omega_{\text {sens. }}$. We assume that all the sensors have the same $r_{\mathrm{s}}, \beta_{\mathrm{sens}}$, and $t_{\mathrm{sens}}$.

The communication interface of the nodes has a transmission range $r_{\mathrm{T}}$ (dimension: [m]), under the constraint that $r_{\mathrm{T}} \gg r_{\mathrm{s}}$. Generally, $r_{\mathrm{T}}$ ranges between $100 \mathrm{~m}$ and $1000 \mathrm{~m}$ (in line-of-sight scenarios). In the remainder of the paper an average value of $250 \mathrm{~m}$ is considered for $r_{\mathrm{T}}$.

We have assumed that only the node detecting the target transmits an alert message to the AP and that there can be only one target in the monitored area at a time. As a result of these assumptions, there are no collisions between the packets transmitted by the wireless devices. $N_{\text {hop }}$ denotes the average number of hops that a packet has to traverse in order to reach the AP. In a surveillance scenario, the Medium Access Control (MAC) protocol must guarantee the lowest energy consumption and the lowest latency in the network. In the rest of the paper, we consider a specific MAC protocol as an example for the derivation of the analytical framework. However, the latter is general in a sense that the optimization techniques are valid for any MAC layer, provided that one recomputes the equations of latency and network lifetime. As an example of a MAC layer, we have chosen the XMAC protocol [3], which is good for low throughput and low latency communications. This random access protocol notably outperforms most of scheduled MAC protocols, according to [4]. The X-MAC protocol and the corresponding parameters, i.e., $S_{\mathrm{d}}, S_{\mathrm{p}}, S_{\mathrm{al}}, \Omega_{\mathrm{T}_{\mathrm{x}}}, \Omega_{\mathrm{R}_{\mathrm{x}}}$, and $\Omega_{\mathrm{s}}$, will be described in Section IV.

To make the derivation of $P_{\mathrm{d}}$ (or, equivalently, of the probability of missed detection $P_{\mathrm{md}}$ ) feasible, we assume the monitored area to be a square with sides of length $d_{\mathrm{s}}$ (dimension: [m]). In this area, $N$ sensors are identically 
and independently deployed in a random fashion under the constraint that their sensing ranges do not overlap. We also assume that the potential targets penetrate the monitored area following a linear and uniform trajectory. Trajectories are characterized by an angle of arrival $\theta$ and a constant target speed $v$ (dimension: $[\mathrm{m} / \mathrm{s}]$ ). Since there is no information about the entrance point, we also assume that the target enters the monitored area from a random point along the perimeter of the monitored surface.

\section{Probability of TARget Missed Detection}

This section first introduces the analytical framework we use for the evaluation of the probability of missed target detection when no sensing duty cycles are considered. Then, we extend this model by allowing the sensing sub-units to be periodically switched off to save energy.

\section{A. Preliminary Background}

Our analytical framework for the evaluation of the probability of target missed detection, considering moving targets, extends the results presented in [5], which we briefly review in the following. For more details about the following derivation, the interested reader is referred to [5]-[7].

In order to detect a target in a squared area with perimeter $L_{0}, N$ sensors are randomly placed over the field of interest. Sensors $s_{i}, i=1, \ldots, N$ have a sensing area of perimeter $L_{i}$. In the case of deterministic deployment, the estimation of $P_{\text {md }}$ would require introducing upper and lower bounds whose computation is left for future work. Assuming that there is no prior knowledge about the direction and the entrance point of the target, the probability $P_{\mathrm{d}}(k)$ that at least $k \geq 1$ sensors detect the target crossing the field of interest is

$$
P_{\mathrm{d}}(k)=1-\sum_{w=0}^{k-1} \sum_{j=1}^{\left|Z_{N, w}\right|} \prod_{i=1}^{\left|z_{j}\right|} q_{z_{j}(i)} \prod_{v=1}^{\left|\bar{z}_{j}\right|}\left(1-q_{\bar{z}_{j}(v)}\right)
$$

where $\left|Z_{N, w}\right|$ denotes the possible $w$-tuples $z_{j}$ of vector $[1, \ldots, N]$, i.e., the possible groups of $w$ sensors that can detect the target at a time, $\bar{z}_{j}$ denotes the complement $(N-w)$-tuple of $z_{j}, q_{i}=q_{z_{j}(i)}=q_{\bar{z}_{j}(i)}=L_{i} / L_{0}$, and $q_{i}$ can be interpreted as $P\{$ Sensor on the trajectory $\}$.

In attempt to simplify the computation, we assume that the sensing shapes are uniform, that is $L_{i}=L \forall i \in\{1, \ldots, N\}$. Equation (1) can be rewritten as

$$
P_{\mathrm{d}}(k)=1-\sum_{i=0}^{k-1}\left(\begin{array}{c}
N \\
i
\end{array}\right) \frac{L^{i}\left(L_{0}-L\right)^{N-i}}{L_{0}^{N}} .
$$

Eq. (1) can also be used to derive the probability of missed detection of a target. In this case, observing that $P_{\mathrm{md}}=P\left(Z_{N, 0}\right)$, i.e., the probability of not detecting any target, and $z_{j}=0, \bar{z}_{j}=\{1, \ldots, N\}$, the probability $P_{\mathrm{md}}$ of missing a target can be written as

$$
P_{\mathrm{md}}=\prod_{i=1}^{N}\left(1-\frac{L_{i}}{L_{0}}\right)
$$

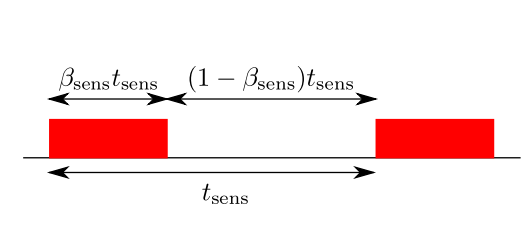

(a)

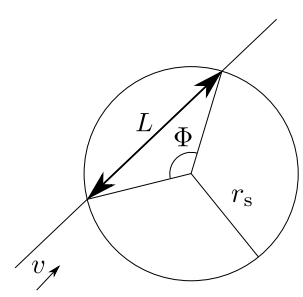

(b)
Figure 1. (a) Logical scheme of the sensing duty cycle and (b) model for the sensing range of a node.

According to the model introduced in Sec. II-B and recalling that all the sensors have the same sensing range, Eq. (2) can be rewritten as follows

$$
P_{\mathrm{md}}=\left(1-\frac{2 \pi r_{\mathrm{s}}}{4 d_{\mathrm{s}}}\right)^{N}
$$

\section{B. Integration of Duty Cycles}

To integrate sleeping duty cycles at the sensing level, we extend the previous model to express $P_{\mathrm{d}}$ or, equivalently, $P_{\mathrm{md}}$, as a function of both the duty cycle $\beta_{\text {sens }}$ and the geometrical configuration of the WSN. The probability of detecting a target is the probability that there is a sensor on the target's trajectory (event denoted as $\mathcal{E}_{\mathrm{SoT}}$ ) and the sensor is active when the target is crossing the sensed area (event denoted as $\mathcal{E}_{\text {det }}$ ). Therefore, the probability that a single sensor detects a target is

$$
P_{\mathrm{d}-1}=P\left\{\mathcal{E}_{\mathrm{SoT}}, \mathcal{E}_{\mathrm{det}}\right\}=P\left\{\mathcal{E}_{\mathrm{det}} \mid \mathcal{E}_{\mathrm{SoT}}\right\} P\left\{\mathcal{E}_{\mathrm{SoT}}\right\} .
$$

According to [5], $P\left\{\mathcal{E}_{\mathrm{SoT}}\right\}$ can be expressed as $2 \pi r_{\mathrm{s}} /\left(4 d_{\mathrm{s}}\right)$.

In order to evaluate $P\left\{\mathcal{E}_{\text {det }} \mid \mathcal{E}_{\mathrm{SoT}}\right\}$, we consider the scheme for the sleeping duty cycle presented in Fig. 1 (a). Since the target arrives with a finite speed $v$, the crossing time is $T_{\text {cross }}=$ $L / v$, where $L$ is a random variable which expresses the length of the intersection between the target's trajectory and the area sensed by a sensor, as shown in Fig. 1 (b). Since there is no information about the arrival of the target, its arrival has been assumed uniformly distributed over the period $t_{\text {sens }}$.

When the sensor is on, any incoming target will be detected. In the case that the sensor is off, i.e., during the interval of duration $\beta_{\text {sens }} t_{\text {sens }}$, the analysis has to be refined. Let $\mathcal{E}_{\text {target }}$ be the event $\{$ The sensor is on at the instant at which the target enters the sensed area\}. Applying the total probability theorem [8], $P\left\{\mathcal{E}_{\mathrm{det}} \mid \mathcal{E}_{\mathrm{SoT}}\right\}$ can then be expressed as

$$
\begin{aligned}
P\left\{\mathcal{E}_{\text {det }} \mid \mathcal{E}_{\text {SoT }}\right\} & =P\left\{\mathcal{E}_{\text {det }} \mid \mathcal{E}_{\text {target }}, \mathcal{E}_{\text {SoT }}\right\} P\left\{\mathcal{E}_{\text {target }} \mid \mathcal{E}_{\text {SoT }}\right\} \\
& +P\left\{\mathcal{E}_{\text {det }} \mid \overline{\mathcal{E}}_{\text {target }}, \mathcal{E}_{\text {SoT }}\right\} P\left\{\overline{\mathcal{E}}_{\text {target }} \mid \mathcal{E}_{\text {SoT }}\right\}(5)
\end{aligned}
$$

where $P\left\{\mathcal{E}_{\text {det }} \mid \mathcal{E}_{\text {target }}, \mathcal{E}_{\text {SoT }}\right\}=1$. Since $\mathcal{E}_{\text {target }}$ and $\mathcal{E}_{\text {SoT }}$ are independent - in fact, the activity cycle of a sensor does not depend on the target-one can write,

$$
P\left\{\mathcal{E}_{\text {target }} \mid \mathcal{E}_{\text {SoT }}\right\}=\mathrm{P}\left\{\mathcal{E}_{\text {target }}\right\}=\int_{0}^{\beta_{\text {sens }} \mathrm{t}_{\text {sens }}} \frac{1}{\mathrm{t}_{\text {sens }}} \mathrm{dt}=\beta_{\text {sens }}
$$

and

$$
P\left\{\overline{\mathcal{E}}_{\text {target }} \mid \mathcal{E}_{\text {SoT }}\right\}=1-\beta_{\text {sens }} .
$$


We are now going to evaluate the last term of Eq. (5). According to the conditioning on $\overline{\mathcal{E}}_{\text {target }}$ and $\mathcal{E}_{\text {SoT }}$, the target arrival time, denoted as $T_{\mathrm{a}}$, is a uniformly distributed random variable over an interval of length $\left(1-\beta_{\text {sens }}\right) t_{\text {sens }}$. To have successful detection, the target must remain in the sensed area until the sensor turns on its sensing device in the following active period. In this case as well, one must distinguish between two cases: (i) $T_{\text {cross }}>\left(1-\beta_{\text {sens }}\right) t_{\text {sens }}$ and (ii) $T_{\text {cross }}<\left(1-\beta_{\text {sens }}\right) t_{\text {sens. }}$. In the former case, each target will be detected, since it remains in the sensed area for a time interval longer than the sleep period. Therefore, in this case $P_{\mathrm{d}}=P\left\{\mathcal{E}_{\mathrm{SoT}}\right\}$. In the latter case, instead, the target will be detected if it enters the sensed area in the last part of the sleep period, so that it will be detected in the following active period.

Let us preliminary observe that, since $T_{\mathrm{a}}$ and $T_{\text {cross }}$ are independent, their joint probability density function (pdf) can be expressed as the product of the marginal pdfs, i.e., $f_{T_{\mathrm{a}}, T_{\text {cross }}}(t, \tau)=f_{T_{\mathrm{a}}}(t) f_{T_{\text {cross }}}(\tau)$. We have already noted that $T_{\mathrm{a}} \sim \operatorname{Unif}[0, c]$, where $c \triangleq\left(1-\beta_{\text {sens }}\right) t_{\text {sens }}$. In order to express $f_{T_{\text {cross }}}(\tau)$, further considerations are required. First of all, $f_{\mathrm{T}_{\text {cross }}}(\tau)=\frac{1}{v} f_{L}(\tau)$. The pdf of the angle $\Phi$, shown in Fig. 1 (b), is needed. The length $L$ of the chord can be expressed as the following function of $\Phi$

$$
L=2 r_{\mathrm{s}} \sin \left(\frac{\Phi}{2}\right) \text {. }
$$

Since $\Phi \sim$ Unif $[0,2 \pi]$, according to the fundamental theorem on page 93 of $[8], f_{L}(\tau)$ becomes

$$
f_{L}(\tau)=\left\{\begin{array}{cc}
\frac{1}{\pi \sqrt{r_{\mathrm{s}}^{2}-\left(\frac{\tau}{2}\right)^{2}}} & \text { if } 0<\tau<2 r_{\mathrm{s}} \\
0 & \text { otherwise. }
\end{array}\right.
$$

Therefore, $f_{T_{\text {cross }}}(\tau)$ can be found and one can finally write

$$
f_{T_{\mathrm{a}}, T_{\text {cross }}}(t, \tau)= \begin{cases}\frac{v}{\pi c \sqrt{r_{\mathrm{s}}^{2}-\left(\frac{v \tau}{2}\right)^{2}}} & \text { if } 0<\tau<\frac{2 r_{\mathrm{s}}}{v}, \\ 0 & \text { otherwise. }\end{cases}
$$

Letting $\mathcal{E}_{1}=\left\{T_{\mathrm{a}}+T_{\text {cross }}>\left(1-\beta_{\text {sens }}\right) t_{\text {sens }}\right\}$, the probability that the target is detected, given that the sensor is in the sleep state when the target enters the sensed area, can be expressed as

$$
P\left\{\mathcal{E}_{\text {det }} \mid \overline{\mathcal{E}}_{\text {target }}, \mathcal{E}_{\text {SoT }}\right\}=P\left\{\mathcal{E}_{1}\right\}=\iint_{\mathcal{D}} f_{T_{\mathrm{a}}, T_{\text {cross }}}(t, \tau) \mathrm{d} t \mathrm{~d} \tau .
$$

where the integration domain $\mathcal{D}$ is shown in Fig. 2 .

Considering the integration domain, two possible cases can be distinguished: (i) $\frac{2 r_{\mathrm{s}}}{v}<c$ and (ii) $\frac{2 r_{\mathrm{s}}}{v}>c$. In the former case, the integration domain $\mathcal{D}$, referring to Fig. 2, reduces to a triangle on the upper left part. $P\left\{\mathcal{E}_{1}\right\}$ can thus be expressed as

$$
P\left\{\mathcal{E}_{1}\right\}=\int_{0}^{\frac{2 r_{\mathrm{s}}}{v}} \int_{c-y}^{c} f_{T_{\mathrm{a}}, T_{\text {cross }}}(t, \tau) \mathrm{d} t \mathrm{~d} \tau=\frac{4 r_{\mathrm{s}}}{\pi c v}
$$

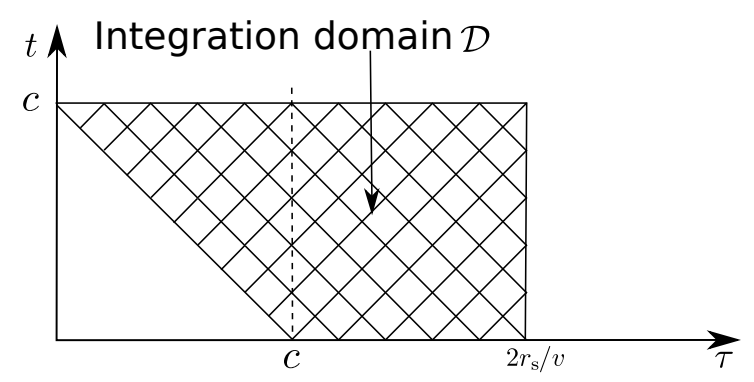

Figure 2. Integration domain for the evaluation of $P\left\{\mathcal{E}_{1}\right\}$.

In the latter case, i.e., when $2 r_{\mathrm{s}} / v>c$, the integration domain is that shown in Fig. 2, the expression of $P\left\{\mathcal{E}_{1}\right\}$ therefore can be rewritten as

$$
\begin{aligned}
P\left\{\mathcal{E}_{1}\right\} & =\int_{0}^{c} \int_{c-y}^{c} f_{T_{\mathrm{a}}, T_{\text {cross }}}(t, \tau) \mathrm{d} t \mathrm{~d} \tau \\
& +\int_{c}^{\frac{2 r_{\mathrm{s}}}{v}} \int_{0}^{c} f_{T_{\mathrm{a}}, T_{\text {cross }}}(t, \tau) \mathrm{d} t \mathrm{~d} \tau q \\
= & \frac{4 r_{\mathrm{s}}-2 \sqrt{4 r_{\mathrm{s}}^{2}-c^{2} v^{2}}}{\pi c v}+1-\frac{2 \mathrm{asin}\left(\frac{c v}{2 r_{\mathrm{s}}}\right)}{\pi}
\end{aligned}
$$

Combining equations (8) and (9) into equation (5), the expression for $P\left\{\mathcal{E}_{\mathrm{det}} \mid \mathcal{E}_{\mathrm{SoT}}\right\}$ can be obtained

$$
P\left\{\mathcal{E}_{\mathrm{det}} \mid \mathcal{E}_{\mathrm{SoT}}\right\}=\beta_{\text {sens }}+\left(1-\beta_{\text {sens }}\right) P\left\{\mathcal{E}_{1}\right\} .
$$

Finally, extending the model in order to take into account that $N$ independent sensors can detect the target, the probability of missed detection becomes

$$
P_{\mathrm{md}}=\left(1-P_{\mathrm{d}-1}\right)^{N}=\left(1-P\left\{\mathcal{E}_{\mathrm{det}} \mid \mathcal{E}_{\mathrm{SoT}}\right\} \frac{2 \pi r_{\mathrm{s}}}{4 d_{\mathrm{s}}}\right)^{N} .
$$

In the case of a heterogeneous sensing model, the derivation is almost identical. The only difference is that we should start the derivation from Eq. (2) instead of from Eq. (3).

\section{Validation}

To validate the analytical framework, we analyze the probability of missed detection $P_{\mathrm{md}}$ through simulations. The reference model for the simulation set-up has been described in Sec. II-B. In order to reduce possible statistical fluctuations, we have considered 1000 different scenarios, corresponding to random node placements within the monitored area. For each scenario, we have considered 1000 target trajectories, characterized by a random entrance point and a random angle of entrance. In Fig. 3, we show $P_{\mathrm{md}}$ as a function of $\beta_{\text {sens }}$, considering different values of $r_{\mathrm{s}}$. The number of nodes is $N=50$ and the speed of the target is $v=15 \mathrm{~m} / \mathrm{s}$. As intuition might suggest, the longer the sensing range, the higher the probability of detecting an incoming target. When the value of $\beta_{\text {sens }}$ becomes small, the target can cross the sensed area during the sleep period of the sensor without being detected, thus increasing the probability of missed detection. In Fig. 3, the simulation results (solid lines) are compared with the theoretical ones (dashed lines). The results show a good agreement between the two models, especially for large 


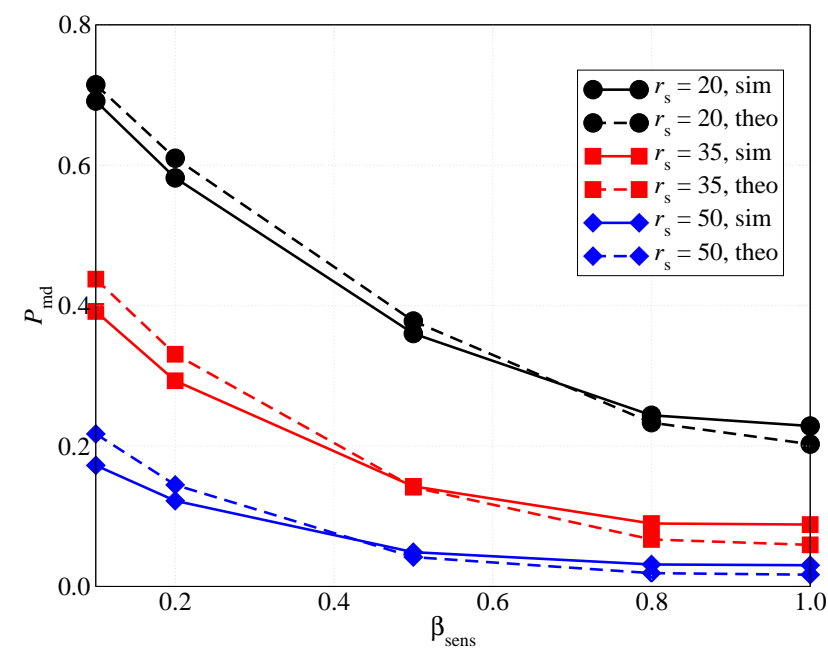

Figure 3. Simulation (solid lines) and analytical (dashed lines) $P_{\mathrm{md}}$ results as functions of the duty cycle $\beta_{\text {sens }}$ considering different sensing ranges. The target enters with speed $v=15 \mathrm{~m} / \mathrm{s}$ a monitored area of $d_{\mathrm{s}}=1000 \mathrm{~m}$ side, where $N=50$ sensors are randomly deployed.

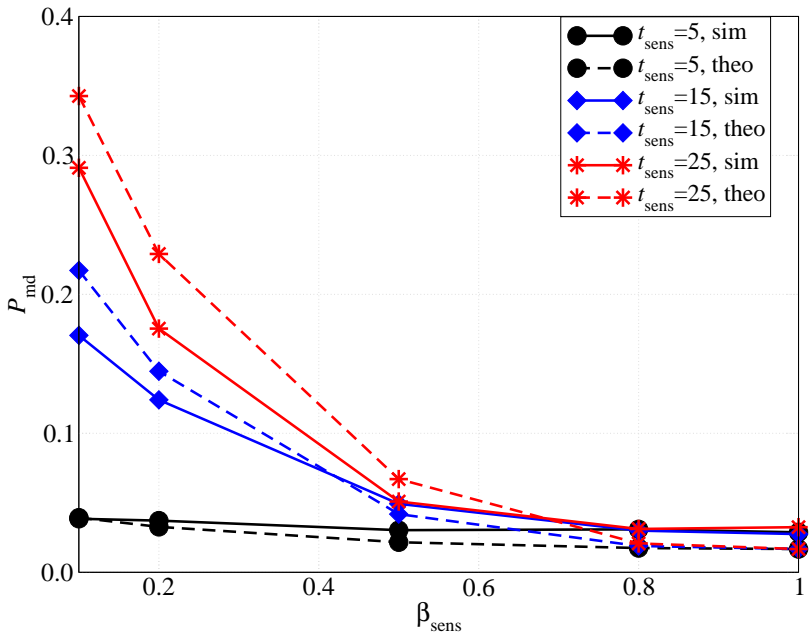

Figure 4. Simulation (solid lines) and analytical (dashed lines) $P_{\mathrm{md}}$ results as functions of the duty cycle $\beta_{\text {sens }}$, considering different period lengths $t_{\text {sens }}$. The size of the area is $d_{\mathrm{s}}=1000 \mathrm{~m}$, in which $N=50$ sensors, with sensing range equal to $r_{\mathrm{s}}=50 \mathrm{~m}$, are randomly placed. The speed of the target is $v=15 \mathrm{~m} / \mathrm{s}$.

values of $r_{\mathrm{s}}$, confirming the validity of the analytical model. The discrepancy between analytical and simulation models, especially for high values of $\beta_{\text {sens }}$, is due to the fact that in the simulations we are considering some target trajectories are crossing the monitored area only marginally. These trajectories are relevant for the computation of $P_{\mathrm{md}}$, even if they are not relevant for realistic scenarios.

In Fig. 4, we evaluate $P_{\mathrm{md}}$ as a function of $\beta_{\text {sens. In all }}$ cases, the target speed $v$ is set to $15 \mathrm{~m} / \mathrm{s}$. In this case, the same considerations carried out for Fig. 3 still hold. When $t_{\text {sens }}$ is sufficiently small, $\beta_{\text {sens }}$ has a limited impact on $P_{\mathrm{md}}$. On the other hand, the larger $t_{\text {sens }}$, the larger the impact of $\beta_{\text {sens. }}$. When $\beta_{\text {sens }}=1$, the sensing period has no impact on the performance of the system. Instead, when $\beta_{\text {sens }}<1$, a larger $t_{\text {sens }}$ leads to a larger $P_{\mathrm{md}}$, because it is more likely a target crosses the sensed area during the sleep phase of the sensor, without being detected. Also in this case, there is a good agreement between the simulation (solid lines) and theoretical (dashed lines) curves. We point out that there is a threshold value of $v$, depending on $r_{\mathrm{s}}, \beta_{\mathrm{sens}}$, and $t_{\text {sens }}$, below which the impact of $\beta_{\text {sens }}$ on $P_{\text {md }}$ becomes negligible and the curves tend to reach a floor. For instance, with $v=1.5 \mathrm{~m} / \mathrm{s}$, a target traverses the sensed areas for a time interval sufficiently long that the sensors along its trajectory will detect it for sure, regardless of their duty cycles.

\section{LATENCY AFTER DETECTION}

In this section, we propose an analytical model for the alert transmission latency, i.e., the delay between the detection instant of the presence of a target by a sensing node and its notification to the sink. In the following, we first derive the per-hop latency, denoted as $D_{1 \text { hop }}$, and then the latency over a multi-hop path. We then verify the validity of this model through experimental measurements on a Crossbow MicaZ testbed.

As mentioned in Sec. II-B, we consider X-MAC [3], a low-power asynchronous MAC-layer protocol for duty-cycled WSNs, for the derivation of the transmission latency. X-MAC uses Low-Power Listening (LPL), or preamble sampling, to enable low-power communications between a sender and a receiver which do not synchronize their wake-up and sleep schedules. Indeed, a sender with data sends a preamble at least as long as the sleep interval of the receiver. This guarantees that the receiver will wake up, detect the preamble, and stay awake for the reception of the data. X-MAC uses a strobed preambling approach in which the sender quickly alternates between sending the packet destination address and a short wait time so that the receiver could potentially abort this process to receive data. This approach allows us to further reduce the energy consumption and per-hop latency in comparison with protocols using long preambles, such as B-MAC [9]. We point out, however, that our approach is valid for any MAC protocol that can be used in a WSN. We chose the X-MAC protocol since it is an improvement on the B-MAC protocol and is more relevant for the application considered.

The average per hop transmission latency can be expressed as

$$
D_{1 \text { hop }}=\frac{\left(1-\beta_{\mathrm{comm}}\right)^{2} t_{\mathrm{comm}}}{2}+S_{\mathrm{p}}+S_{\mathrm{al}}+S_{\mathrm{d}}
$$

where $\beta_{\text {comm }}$ is the (normalized) communication duty cycle over the period $t_{\text {comm }}$, and $S_{\mathrm{p}}, S_{\mathrm{al}}$, and $S_{\mathrm{d}}$ are durations (dimension: [s]) of the strobed preamble, the acknowledgement of the preamble, and the alert packet, respectively. Considering the status of the receiving node, i.e., if the communication subsystem is either turned on or off, the probability that a node begins its transmission when the receiving node is on is $\beta_{\text {comm }}$ and the associated latency is $S_{\mathrm{p}}+S_{\mathrm{al}}+S_{\mathrm{d}}$. On the other hand, the probability that a node is off is $1-\beta_{\text {comm. }}$. We evaluate $D_{1 \text { hop }}$ simply as the average between the worst and best cases. The best case is when a node starts transmitting exactly when the receiving node starts its LPL operations, so that the packet is transmitted after $S_{\mathrm{p}}+S_{\mathrm{al}}+S_{\mathrm{d}}$. In the worst case, the 
transmitting node waits for the entire duration of the sleep interval. In addition, since the receiving node must receive an entire preamble before sending the acknowledgment message, the worst case takes into account that two transmissions of the preamble may be required in order to start the communication. In this case, the latency introduced by the transmission is $\left(1-\beta_{\text {comm }}\right) t_{\text {comm }}+\left(S_{\mathrm{p}}+S_{\mathrm{al}}\right)+S_{\mathrm{d}}$. Scaling this term by the probability that the receiver is off, averaging the best and the worst cases and adding the latency, related to the case with the receiver on, scaled by its probability of being on, equation (12) is obtained.

Considering a multi-hop path, the average global latency can be expressed as follows

$$
D=D_{1 \text { hop }} N_{\text {hop }}
$$

where $N_{\text {hop }}$ denotes the average number of nodes that the alert message traverses to reach the sink.

Note that the model relies on a collision-free transmission of the alert as we assume a single target detection scenario with low arrival rate. Therefore, there is only one sending node at a time that does not experience packet losses and retransmissions. We also neglect the processing time of the packets.

In order to verify the analytical model of the multi-hop alert transmission latency, we have run a set of experimental tests with a testbed of 4 Crossbow MicaZ nodes deployed in a chain topology. The first node injects a packet every 2 s. Each subsequent node forwards the packet to its neighbor until it reaches the last node, which reverses the transmission's direction. We have measured the round trip time setting $N_{\text {hop }}$ from 2 to 6 , and $\beta_{\text {comm }}$ to either $0.067,0.1$ or $0.2 .^{1}$ For each pair of values, we compute the average latency as the mean round-trip time of 100 samples, along with the $95 \%$ confidence interval. In Fig. 5, a comparison between the theoretical and experimental results is shown. The curves are quite close, even though a gap appears when $\beta_{\text {comm }}$ becomes lower and the duration of the sleep interval increases. This may be due to the unavoidable alignment of sleep schedules on the packet's way back, which tends to reduce the latency for large values of sleep interval durations, i.e., small values of $\beta_{\text {comm. }}$. In the context of the reference scenario, the experiments show the validity of the analytical model, so that the latency can be approximated as $D \simeq N_{\text {hop }}\left(1-\beta_{\text {comm }}\right) t_{\text {comm }} / 2$.

\section{Average Network Lifetime}

As nodes operate on batteries, the way they consume energy directly impacts the lifetime of the surveillance system. To take this into account, we now propose a simple energy model for the engineering toolbox.

The energy consumption of nodes can be roughly given by the sum of the energies consumed by its hardware components. For the sake of simplicity, we only integrate in the energy model contributions from the sensing sub-unit and the radio

\footnotetext{
${ }^{1}$ These values of $\beta_{\text {comm }}$ correspond to a static active period of $8 \mathrm{~ms}$ and $t_{\text {comm }}$ respectively equal to $120 \mathrm{~ms}, 80 \mathrm{~ms}$ and $40 \mathrm{~ms}$, respectively.
}

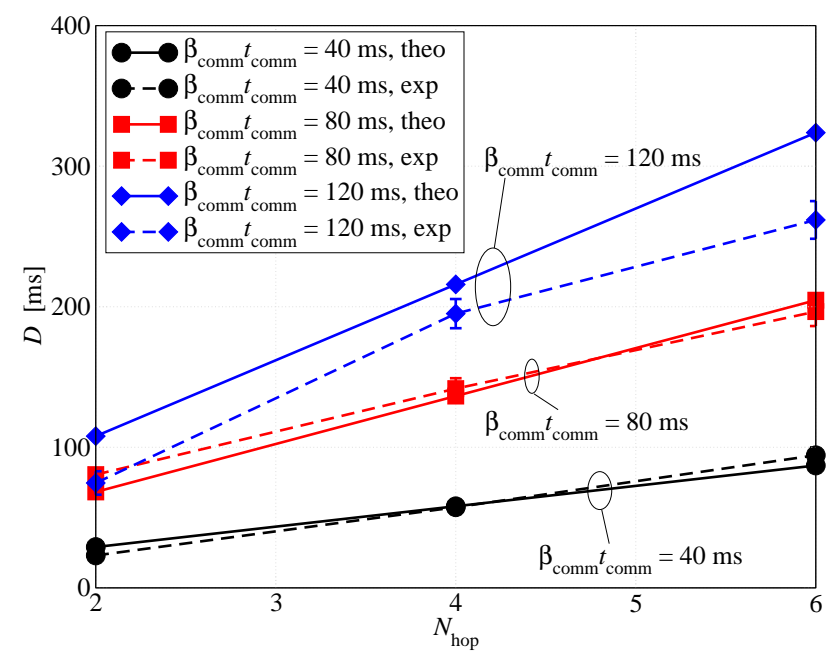

Figure 5. Latency as a function of the number of hops traversed by a packet. Both (i) experimental (dashed lines) and (ii) theoretical results (solid lines) are presented.

transceiver. We also define the network lifetime as the time needed for the average residual energy $E_{\mathrm{r}}$ to be lower than a threshold value $E_{\mathrm{th}}$.

To derive an expression for the network lifetime, we evaluate the energy consumed after given interval. The average residual energy $E_{\mathrm{r}}$ at an instant $t$ can be expressed as

$$
E_{\mathrm{r}}(t)=N E_{\mathrm{i}}-N \Omega_{\mathrm{tot}} t
$$

where $E_{\mathrm{i}}$ is the initial energy of a node and $\Omega_{\mathrm{tot}}$ is the total power consumed by sensing and communication operations.

According to the description of the X-MAC protocol in Section IV, there are four possible states for a node: (i) transmission, (ii) reception, (iii) sleep, and (iv) LPL, with corresponding power consumptions denoted as $\Omega_{\mathrm{T}}, \Omega_{\mathrm{R}}, \Omega_{\mathrm{s}}$, and $\Omega_{\mathrm{LPL}}$, respectively. $\Omega_{\text {tot }}$ can then be computed as follows

$$
\Omega_{\text {tot }}=\Omega_{\text {sensing }}+\Omega_{\mathrm{LPL}}+\left(\Omega_{\mathrm{R}}+\Omega_{\mathrm{T}}\right) P_{\mathrm{d}} N_{\text {target }}
$$

where: $\Omega_{\text {sensing }}$ is the power consumption associated with the sensing device in the activity period of duration $t_{\text {sens }} ; \Omega_{\mathrm{LPL}}$ is the power required when performing the LPL operations (over a period of duration $t_{\text {comm }}$ ); $\Omega_{\mathrm{R}}$ is the power used by a node to receive a packet; $\Omega_{\mathrm{T}}$ is the power used to transmit an alert packet; $P_{\mathrm{d}}$ is the target detection probability; and $N_{\text {target }}$ is the number of times that a target appears during a reference period. The expected power to send a packet can be expressed as

$$
\begin{array}{r}
\Omega_{\mathrm{T}}=\left[\Omega_{\mathrm{T}_{\mathrm{x}}} S_{\mathrm{d}}+\frac{\left(1-\beta_{\text {comm }}\right) t_{\text {comm }}}{2\left(S_{\mathrm{p}}+S_{\mathrm{al}}\right)} .\right. \\
\left.\cdot\left(\Omega_{\mathrm{T}_{\mathrm{x}}} S_{\mathrm{p}}+\Omega_{\mathrm{R}_{\mathrm{x}}} S_{\mathrm{al}}\right)\right] \frac{N_{\mathrm{hop}}}{N t_{\mathrm{comm}}}
\end{array}
$$

where the first additive term, i.e., $\Omega_{\mathrm{T}_{\mathrm{x}}} S_{\mathrm{d}}$, is the energy spent to transmit a packet, whereas the last additive term expresses the energy consumption due to the periodic preamble transmission in order to notify the receiving node of a packet arrival. As in Section IV, since nodes are not synchronized, we assume the average number of preamble transmissions to be the mean between best and worst cases. The term $N_{\text {hop }} / N$ is introduced 
owing to the fact that only a subset of the $N$ sensors is used to relay the alert message to the AP.

The expected power to receive a packet can be expressed as

$$
\Omega_{\mathrm{R}}=\frac{\left[\Omega_{\mathrm{R}_{\mathrm{x}}} S_{\mathrm{d}}+\left(\Omega_{\mathrm{R}_{\mathrm{x}}} S_{\mathrm{p}}+\Omega_{\mathrm{T}_{\mathrm{x}}} S_{\mathrm{al}}\right)\right]}{t_{\mathrm{comm}}} \frac{N_{\text {hop }}}{N}
$$

where $\Omega_{\mathrm{R}_{\mathrm{x}}} S_{\mathrm{d}}$ is the energy spent to receive a packet and $\Omega_{\mathrm{R}_{\mathrm{x}}} S_{\mathrm{p}}+\Omega_{\mathrm{T}_{\mathrm{x}}} S_{\mathrm{al}}$ is the energy spent for the reception of the preamble and the transmission of the acknowledgment message. Since in the scenario considered there is no information about the position of the AP, the average number of hops has been determined by calculating, using the Dijkstra algorithm, the shortest path for each node to reach every other node in the network. Given that $r_{\mathrm{T}}$ is around $250 \mathrm{~m}$, the value of $N_{\text {hop }}$ is around 2.7. However, since it is reasonable to consider a integer value for $N_{\text {hop }}$, this value has been rounded to 3 .

The power associated with the LPL operations can be expressed as

$$
\Omega_{\mathrm{LPL}}=\Omega_{\mathrm{R}_{\mathrm{x}}} \beta_{\text {comm }}+\Omega_{\mathrm{s}}\left(1-\beta_{\text {comm }}\right)-\Gamma_{\mathrm{T}_{\mathrm{x}}}-\Gamma_{\mathrm{R}_{\mathrm{x}}}
$$

where $\Gamma_{\mathrm{T}_{\mathrm{x}}}$ and $\Gamma_{\mathrm{R}_{\mathrm{x}}}$ are two corrective terms. In particular, equations (16), (17), and (18) are evaluated over a period $t_{\text {comm. }}$. However, during normal operations the node either performs LPL operations or transmits/receives a packet. $\Gamma_{\mathrm{T}_{\mathrm{x}}}$ and $\Gamma_{R_{x}}$ are used to refine the power consumption due to LPL operations. In fact, the LPL and also the transmission and reception intervals overlap for short intervals, so that without these two terms the power consumption budget would be higher than the correct one. In particular, $\Gamma_{\mathrm{T}_{\mathrm{x}}}$ can be expressed as

$$
\begin{aligned}
\Gamma_{\mathrm{T}_{\mathrm{x}}}=\left[\Omega_{\mathrm{s}}[\right. & \left.\frac{\left(1-\beta_{\text {comm }}\right) t_{\text {comm }}}{2}+S_{\mathrm{d}}+S_{\mathrm{al}}\right] \\
& \left.+\Omega_{\mathrm{R}_{\mathrm{x}}} S_{\mathrm{p}}\right] P_{\mathrm{d}} N_{\text {target }} \frac{N_{\text {hop }}}{N t_{\text {comm }}}
\end{aligned}
$$

whereas $\Gamma_{\mathrm{R}_{\mathrm{x}}}$ can be expressed as

$$
\begin{array}{r}
\Gamma_{\mathrm{R}_{\mathrm{x}}}=\left[\left(S_{\mathrm{al}}+S_{\mathrm{d}}\right) \Omega_{\mathrm{s}}+\Omega_{\mathrm{R}_{\mathrm{x}}} S_{\mathrm{p}}\right] \\
\cdot P_{\mathrm{d}} N_{\text {target }} \frac{N_{\mathrm{hop}}}{N t_{\text {comm }}} .
\end{array}
$$

The $\Gamma_{\mathrm{T}_{\mathrm{x}}}$ term must take into account the fact that, during transmission operations, such as periodic preamble transmission over an interval of duration $\left(1-\beta_{\text {comm }}\right) t_{\text {comm }} / 2$, packet transmission and acknowledgment reception, a node would normally be in the sleep state, whereas during the transmission of the preamble, that will be acknowledged by the receiving node, a node would normally be in the reception state. Thus, $\Gamma_{\mathrm{T}_{\mathrm{x}}}$ is a correction factor, since otherwise the energy consumed by the node with this model would be higher than the real value because reception and transmission operations overlap with normal LPL operations for a period. Similar considerations are addressed by the $\Gamma_{\mathrm{R}_{\mathrm{x}}}$ term. In fact, when a node is waiting for the acknowledgment window to transmit an acknowledgment message, receiving the preamble and transmitting a packet, according to the LPL operations, it

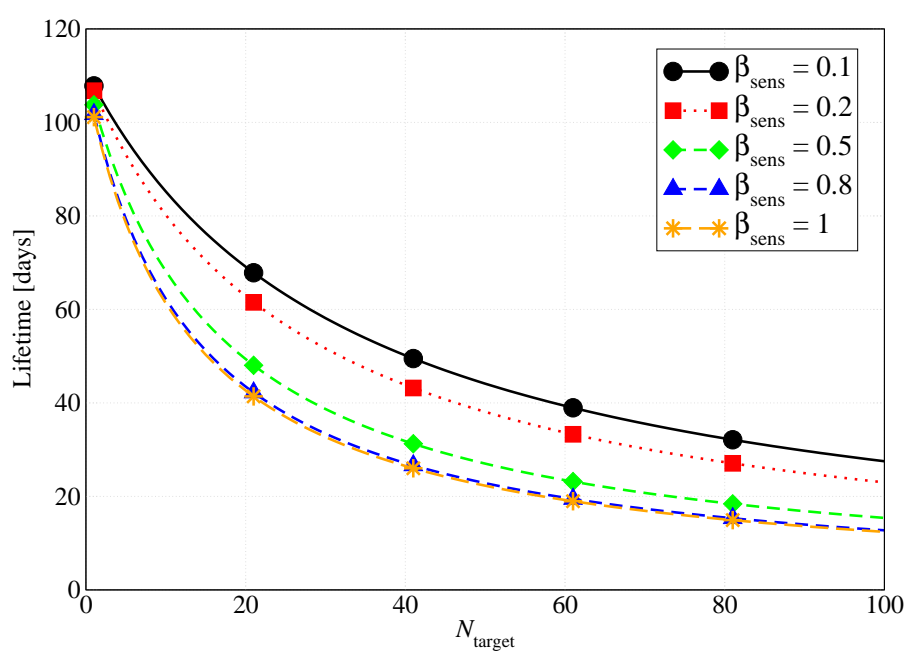

Figure 6. Expected lifetime, expressed in days of activity of the network, as a function of the arrival rate of the target during a day $\left(N_{\text {target }}\right)$.

would normally be in the sleep state. The same considerations come into play when a node is receiving the preamble, since it would normally be in the receiving state for the LPL operations.

Finally, the power consumed during sensing operations can be expressed as

$$
\Omega_{\text {sensing }}=\beta_{\text {sens }} \Omega_{\text {sens }} \text {. }
$$

Introducing the expressions in (19) and (20) into (18) and the expressions in (16), (17), (18), and (21) into (14), it is possible to derive an expression for the energy consumption which depends on both sensing and communication parameters.

In order to derive an expression for the network lifetime $\mathcal{L}$, equation (14) can be rewritten as

$$
\mathcal{L}=\frac{N E_{\mathrm{i}}-E_{\mathrm{th}}}{N \Omega_{\mathrm{tot}}}
$$

where $E_{\mathrm{th}}$ is a given residual energy threshold, which can be used to model the physical behavior of a node.

In Fig. 6, the lifetime [given by equation (22)] of a randomly generated network is shown, as a function of the target arrival rate, for various values of $\beta_{\text {sens }}$. In this case, for simplicity, we have considered $E_{\mathrm{th}}=0$, but our framework can be applied for any value of $E_{\mathrm{th}}$. When the target arrival rate is low, the energy depletion of the battery is mainly due to the LPL and sensing operations. With an increase in the target arrival rate, the impact of the transmission and reception terms becomes significant and tends to dominate the LPL and sensing terms. Surprisingly, the duty cycle of the sensing device has an impact on the transmission and reception terms. In fact, according to the results presented in Sec. III-B, the larger $\beta_{\text {sens }}$, the higher the probability of detecting a target and, consequently, the larger the number of transmissions from a sensor to the AP.

\section{SySTEM ENGINEERING}

This section illustrates the use of the engineering toolbox presented in the paper. First, we investigate the space of 


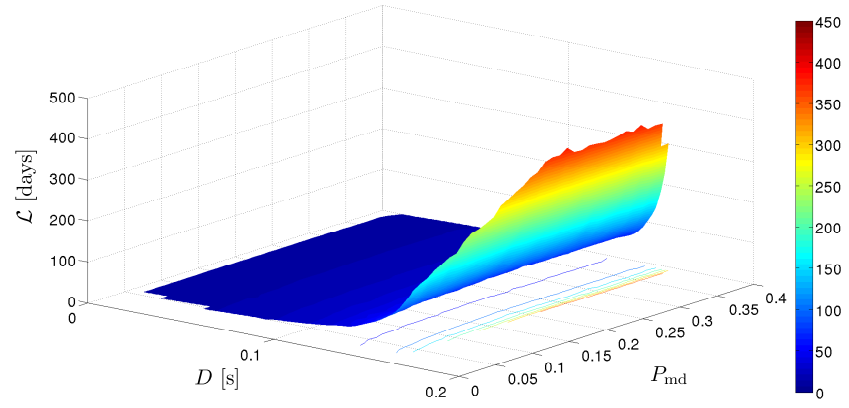

Figure 7. Joint optimization of $D, P_{\mathrm{md}}$, and $\mathcal{L}$. No constraints are imposed.

optimum configurations from the perspective of the tradeoffs faced by the kind of WSN under consideration. Then, we present an application case in which a given surveillance system is optimally configured to maximize the sustainability of the network, i.e., its lifetime $\mathcal{L}$, for certain quality of service requirements in terms of probability of missed detection $\left(P_{\mathrm{md}}\right)$ and latency for alert transmission to the AP $(D)$.

The engineering toolbox consists of the three expressions derived in previous sections for $P_{\mathrm{md}}, \mathcal{L}$, and $D$. Since equations (11) and (22) are not linear, standard optimization techniques for linear programming cannot be used. However, the three equations identify a convex set, which makes gradientbased optimization techniques feasible [10]. When two or more functions need to be optimized, we use multi-objective optimization techniques, which allow us to simultaneously optimize conflicting objective functions, subject to certain (if any) constraints. Throughout this section, the target arrival rate $N_{\text {target }}$ is fixed at 10 targets per day.

\section{A. System Trade-offs}

This subsection investigates the space of optimum configuration parameters (number of nodes $N$ and duty cycles $\beta_{\text {comm }}$ and $\beta_{\text {sens }}$ ), using unconstrained optimization, to highlight the trade-offs faced by the WSN considered. In order to solve the optimization problem, we chose a Pareto-compliant ranking method based on evolutionary techniques, namely the Non-dominated Sorting Genetic Algorithm-II (NSGA-II) [11]. Fig. 7 shows the result of the joint optimization of the three objective functions, namely latency, lifetime, and probability of missed detection. These results show that the longer the latency or the higher the probability of missed detection, the longer the lifetime. In particular, when latency is short, i.e., the nodes must have communication interfaces on for a large portion of the interval $t_{\text {comm }}$, the lifetime is affected mainly by the duty cycle $\beta_{\text {comm }}$, and the impact of $\beta_{\text {sens }}$ is negligible. On the other hand, for long latency values the nodes can keep $\beta_{\text {sens }}$ low, and, consequently, the impact of $P_{\mathrm{md}}$ on lifetime is far more pronounced.

The important outcome of the results in Fig. 7 is that it provides details about the optimal network configuration, since tuning the network with the parameters derived from the execution of the NSGA-II algorithm guarantees that the network performance is not biased toward any one of the

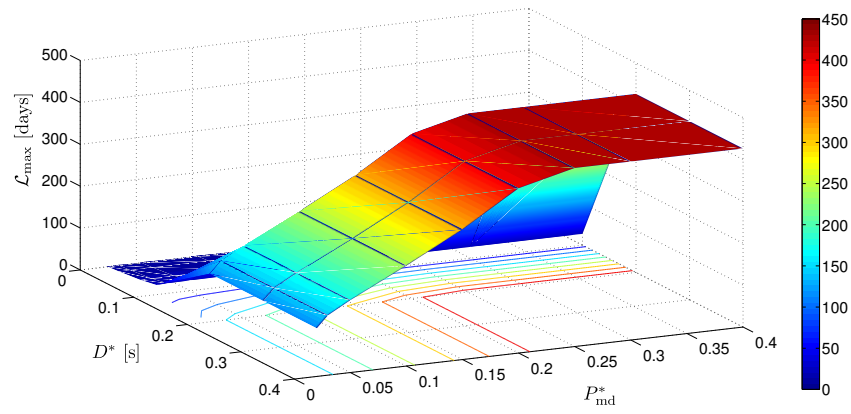

Figure 8. Lifetime maximization under probability of missed detection $\left(P_{\mathrm{md}}^{*}\right)$ and latency $\left(D^{*}\right)$ constraints.

performance indicators. $^{2}$

\section{B. An Application Case}

This subsection investigates a realistic use case where one wants to maximize the lifetime of a given WSN according to given constrained maximum values of $D$ and $P_{\mathrm{md}}$, denoted as $D^{*}$ and $P_{\mathrm{md}}^{*}$, respectively. This use case consists of the optimization of a single-objective function, given constraints on the two other functions. The optimal parameters can be evaluated using single-objective convex programming techniques. The fmincon Matlab toolbox or the optimization approach presented in Section VI-A can be used. In Fig. 8, the lifetime of the network has been maximized under the constraints $P_{\mathrm{md}}<P_{\mathrm{md}}^{*}$ and $D<D^{*}$. The optimization has been carried out considering several values of $P_{\mathrm{md}}^{*}$ and $D^{*}$. In particular, for each pair of values considered, we have evaluated the maximum lifetime that can be obtained. Of course, the more stringent the requirements on $D$ and $P_{\mathrm{md}}$, the shorter the longest achievable lifetime. In fact, in the case of small values of $P_{\mathrm{md}}^{*}$ and $D^{*}$, a sensor must keep its sensing interface on (to minimize $P_{\mathrm{md}}$ and $D$ ) and its communication interface on (to minimize $D$ ) for a large portion of the period, so energy consumption increases and the lifetime decreases. On the other hand, when the requirements are less stringent, the sensing and communication interfaces can be switched off for a longer portion of the period, and, consequently, the lifetime increases.

Focusing on the shape of the surface, generated by interpolation of the simulation results, it is possible to understand the contribution of both sensing and communication operations. For a given value of $P_{\mathrm{md}}^{*}$, one can observe that the shape of the projection of the network lifetime over the $D^{*}-\mathcal{L}_{\max }$ plane remains the same. In the case of the joint optimization of the three metrics, the maximum latency is limited to $0.15 \mathrm{~s}$, and this is why the $D$ axis ranges between $0 \mathrm{~s}$ and $0.2 \mathrm{~s}$. In this case, instead, the $D^{*}$ and $P_{\mathrm{md}}^{*}$ axes refer to the constraints required by a given application and, therefore, their range is within a wider interval. When the latency requirements are stringent, the lifetime is short. Obviously, if the latency requirements are relaxed, the lifetime increases towards a

\footnotetext{
${ }^{2}$ The $D$ axis ends at $0.2 \mathrm{~s}$ because the maximum latency value, which is associated with the smallest value of $\beta_{\text {comm }}$ (i.e., 0.0025 ) allowed by the $\mathrm{X}$-MAC protocol, is $0.15 \mathrm{~s}$ over the average 3-hop path considered.
} 
saturation value which depends on $P_{\mathrm{md}}^{*}$. A short latency corresponds to a short network lifetime, since (i) the power consumed during reception is several orders of magnitude larger than the power consumed in the sleep phase and (ii) the communication interface of each node must be on for a large portion of the communication period $t_{\text {comm }}$ in order to ensure that a packet is delivered to the AP in a short time. The impact of the latency on $\mathcal{L}_{\max }$ becomes negligible, i.e., the lifetime saturates, with respect to $D^{*}$, when $D^{*} \simeq 0.15 \mathrm{~s}$. In fact, this value corresponds to the minimum allowed value of $\beta_{\text {comm }}$ that guarantees that a preamble is correctly received in order for a communication to start. Therefore, larger values of $D^{*}$ have no impact on the latency, since lower values of $\beta_{\text {comm }}$ cannot be selected.

Focusing on the $P_{\mathrm{md}}^{*}-\mathcal{L}_{\max }$ plane, it turns out that the power consumption during sensing is basically negligible when the latency requirements are limited. In fact, the power consumption associated with sensing operations is one order of magnitude lower than that related to reception operations. When the maximum tolerable latency becomes longer than $0.15 \mathrm{~s}$, the power consumption related to the communication interface remains constant and the lifetime is affected only by the sensing power consumption. In this case, the lower $P_{\mathrm{md}}^{*}$ (i.e., the more stringent the constraint on the probability of missed detection), the higher the energy consumption, since a sensor must be on for a longer interval.

Unlike the case with joint optimization (with three objective functions) considered in Fig. 7, in this case the parameters associated with the given values of $D^{*}$ and $P_{\mathrm{md}}^{*}$ may not lead to optimum network configuration, even if the lifetime is maximized. In fact, the solution obtained with a single objective minimization may lead to a "dominated" network configuration, i.e., a solution where one performance indicator is dominating over the others. This approach, however, guarantees that the network parameters are correctly configured and that no energy is wasted by the nodes during their communication and sensing operations.

\section{CONCLUding REMARKS}

This paper has addressed the problem of engineering energy-efficient target detection applications using Wireless Sensor Networks (WSNs). In particular, we have first proposed an engineering toolbox which contains a set of models for describing the probability of missed detection, the alert transmission latency, and the energy consumption under the assumption of random node deployment. By leveraging this toolbox, we have then characterized the trade-offs faced by WSNs with respect to energy consumption and quality of service, in terms of detection capabilities and latency. Finally, we have illustrated the use of the toolbox to optimally configure a given WSN for a variety of quality of service requirements. As such, the engineered toolkit gives the possibility to an operator to set up efficiently an unattended WSN for a wide range of scenarios like counting applications with poor latency requirements (e.g., counting animals in a given area using passive infra-red sensing), as well as live monitoring applications with a strong latency and medium $P_{\text {md }}$ requirements (e.g., tracking emergency or panic situations in a public subway, using audio sensing).

Further works along these lines include extensions of the engineering toolbox to encompass (i) deterministic node deployment, addressing, for instance, physical constraints imposed by the surface morphology, (ii) non-ideal sensing and transmission ranges, using multiple sensing modalities, and (iii) exhaustive energy models for complex sensor nodes, in order to improve the overall accuracy with respect to real operating conditions.

\section{ACKNOWLEDGEMENTS}

This work has been partly funded by the French ANR SensLab project and the European ITEA GEODES project. We also would like to thank Prof. Loukas Lazos from University of Arizona for his useful comments.

\section{REFERENCES}

[1] T. He, S. Krishnamurthy, L. Luo, T. Yan, L. Gu, R. Stoleru, G. Zhou, Q. Cao, P. Vicaire, J. A. Stankovic, T. F. Abdelzaher, J. Hui, and B. Krogh, "Vigilnet: An integrated sensor network system for energyefficient surveillance," ACM Trans. Sen. Netw., vol. 2, no. 1, pp. 1-38, February 2006.

[2] J. Jeong, Y. Gu, T. He, and D. Du, "VISA: Virtual Scanning Algorithm for Dynamic Protection of Road Networks," in Proc. of 28th IEEE Conference on Computer Communications (INFOCOM 09), Rio de Janeiro, Brazil, April 2009.

[3] M. Buettner, G.V. Yee, E. Anderson, and R. Han, "X-mac: a short preamble mac protocol for duty-cycled wireless sensor networks," in Proc. of 4th ACM Int. Conf. on Embedded networked sensor systems (SenSys '06), Boulder, CO, USA, November 2006, pp. 307-320.

[4] K. Klues, G. Hackmann, O. Chipara, and C. Lu, "A component-based architecture for power-efficient media access control in wireless sensor networks," in Proc. of the 5th ACM Int. Conf. on Embedded networked sensor systems (SenSys '07), Sidney, Australia, November 2007, pp. $59-72$.

[5] L. Lazos, R. Poovendran, and J. A. Ritcey, "Analytic evaluation of target detection in heterogeneous wireless sensor networks," ACM Trans. Sensor Networks, vol. 5, no. 2, pp. 1-38, March 2009.

[6] L. A. Santalò and M. Kac, Integral geometry and geometric probability, Cambridge University Press, Cambridge, UK, 2004.

[7] H. Solomon, Geometric probability, SIAM, Philadelphia, PA, USA, 1978.

[8] A Papoulis, Probability, Random Variables and Stochastic Processes, McGraw-Hill, New York, NJ, USA, 1991.

[9] J. Polastre, J. Hill, and D. Culler, "Versatile Low Power Media Access for Wireless Sensor Networks," in Proc. of 2nd ACM Int. Conf. on Embedded networked sensor systems (SenSys '04), Baltimore, MD, USA, November 2004, pp. 95-107.

[10] D. Bertsimas and J.N. Tsitsiklis, Introduction to linear optimization, Athena Scientific, Belmont, MA, USA, 1997.

[11] K. Deb, S. Agrawal, A. Pratap, and T. Meyarivan, "A fast elitist nondominated sorting genetic algorithm for multi-objective optimization: NSGA-II," in Proc. of the 6th Int. Conf. on Parallel Problem Solving From Nature (PPSN VI), Paris, France, September 2000, pp. 849-858. 